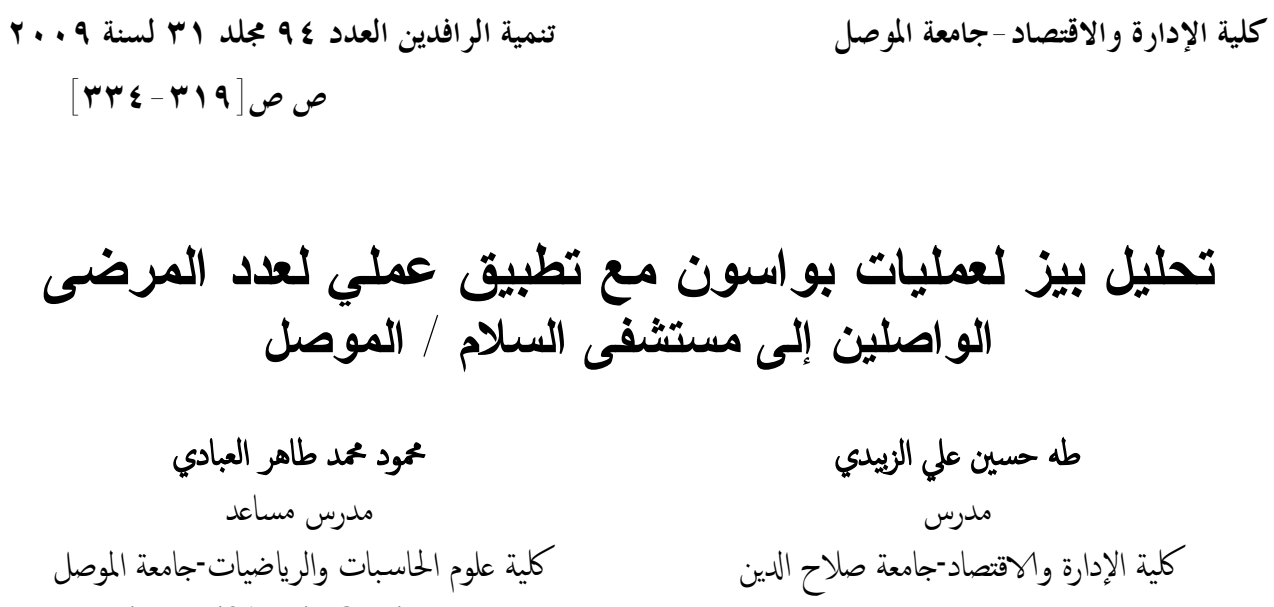

كلية الإدارة والاقتصاد-جامعة صلاح الدين

Mahmood81_tahr@yahoo.com

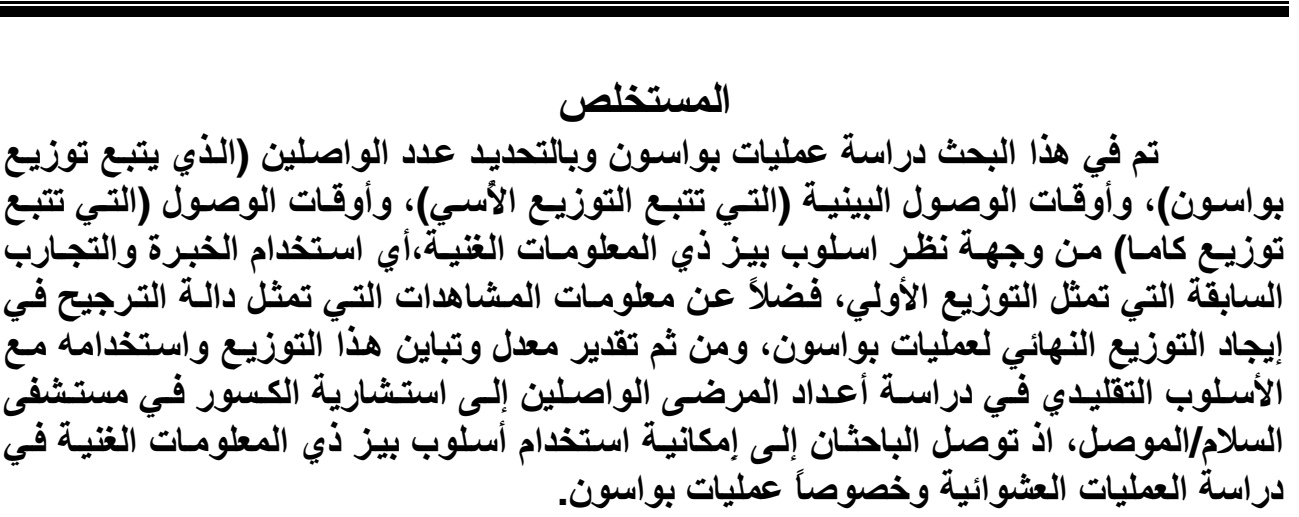

\title{
Bayes's Analysis For Boisson Processes With Practical Application In Al-Salam Hospital/Mosul
}

Taha H. Al-Zubaidy

Lecturer

University of Salah Aldin

\author{
Mahmood M. Al-Abady \\ Assistant Lecturer \\ University of Mosul
}

\begin{abstract}
This paper studies the Boisson process, precisely the number of arrivals (according to the Boisson distribution) and inter - arrival times (according to the exponential distribution ) and arrival times (according to the gamma distribution) from the point view of bayes statistics which is posterior in information, i.e., using the past experiences which represents the prior distribution, besides the direct data, which represents likelihood function in founding posterior distribution of Boisson process, then estimate the mean and the variance of this distribution, and using it with the classic way in studying the number of patients
\end{abstract}

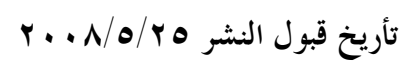

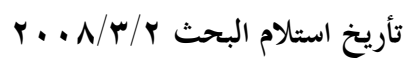


arriving to the consultative clinic of fracture in al-Salam hospital/Mosul. The researchers concluded that bayes statistics, which is posterior in information, could be used studying stochastic process, especially the Boisson process.

[6] 16

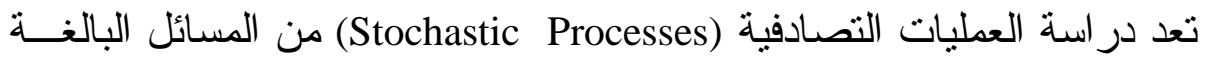

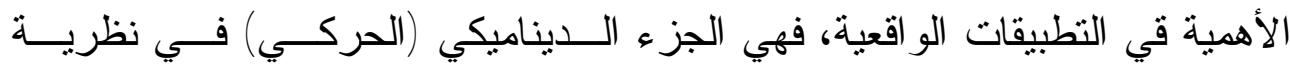

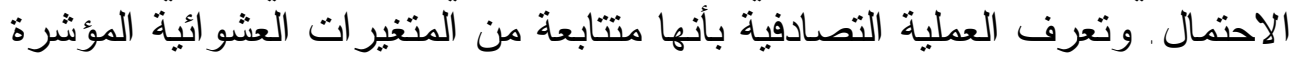

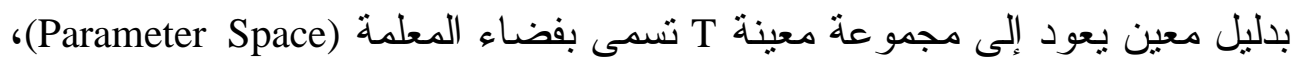

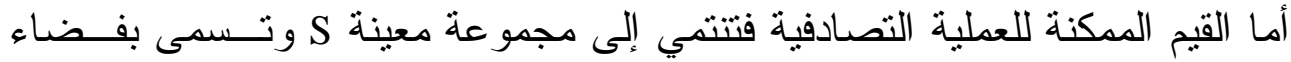

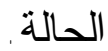

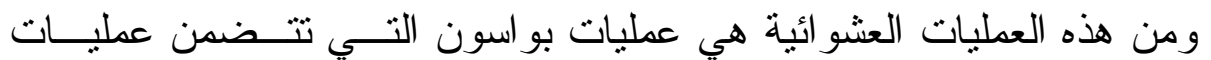

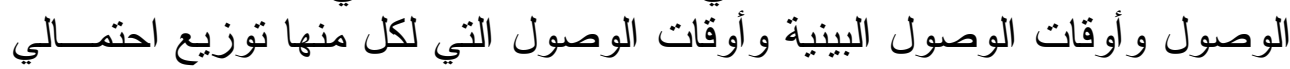
معين تخضع له اله.

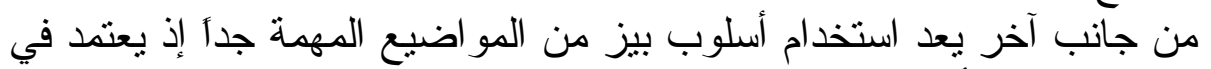

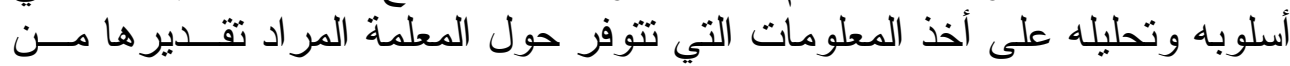

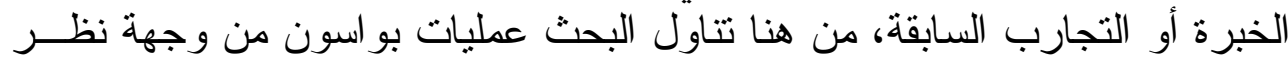

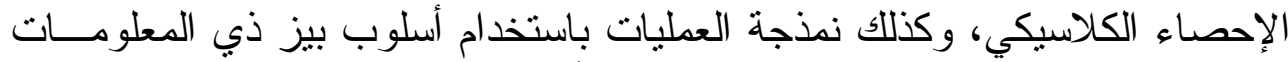

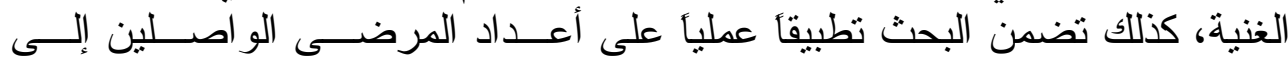

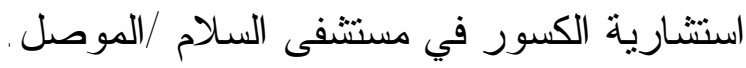

r. r عمليات بواسون (Poisson Process)

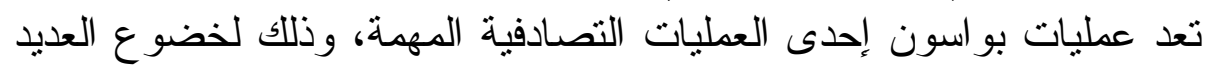

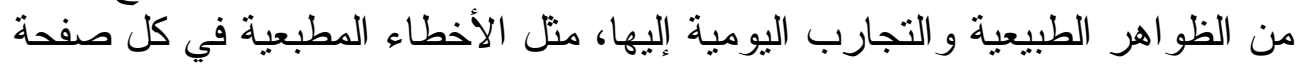

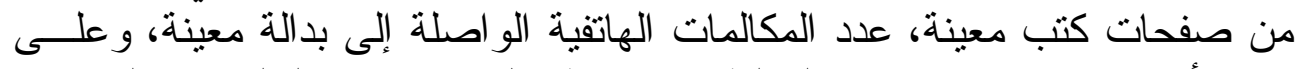

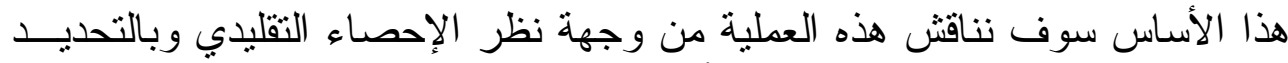

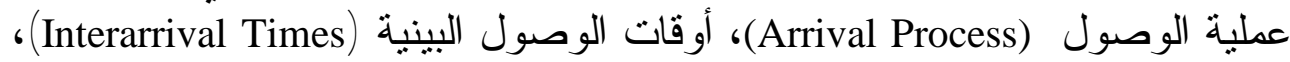

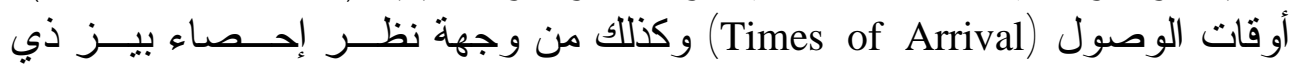

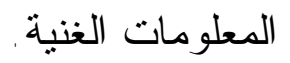

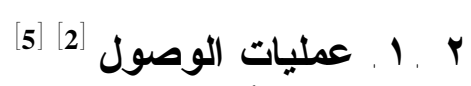

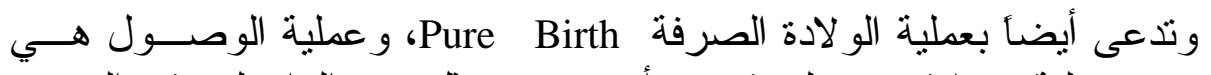

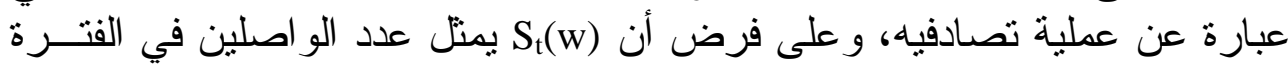

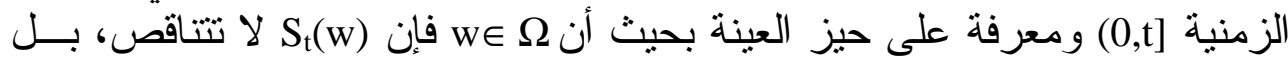

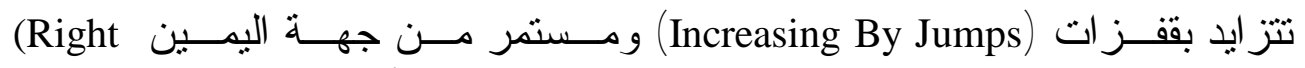
(Continuous)

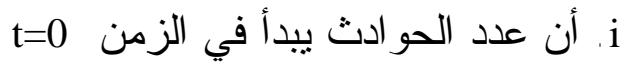




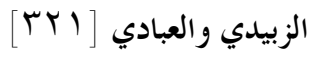

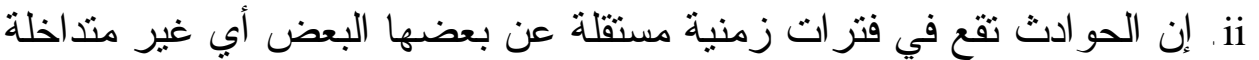

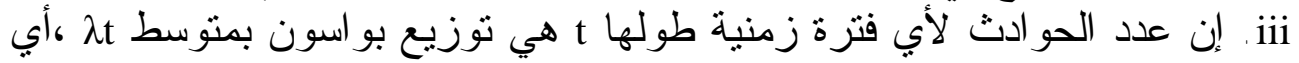

$$
\begin{aligned}
& p\left(s_{t+k}-s_{t}=n\right)=\frac{\mathrm{e}^{-\lambda t}(\lambda t)^{n}}{n !} \quad \mathrm{n}=0,1,2,3, \ldots
\end{aligned}
$$

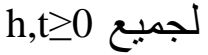

$$
\begin{aligned}
& \text { وبما أن عدد الو اصلين رمزنا له بـ }
\end{aligned}
$$

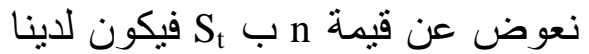

$$
\begin{aligned}
& p\left(s_{t}\right)=\frac{\mathrm{e}^{-\lambda t}(\lambda t)^{S_{t}}}{S_{t} !} \quad \mathrm{S}_{\mathrm{t}}=0,1,2,3,
\end{aligned}
$$

ومعدل وتباين هذا التوزيع لعدد الو اصلين كما هو معلوم

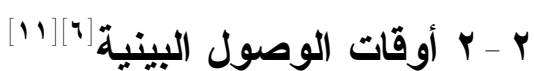

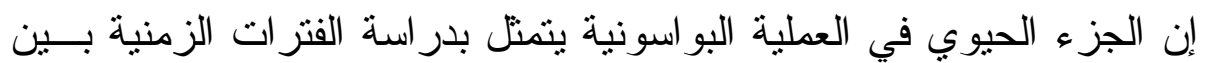

الحو ادث، فإذا فرضنا أن العملية التصادفية

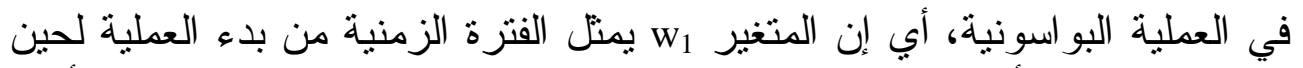

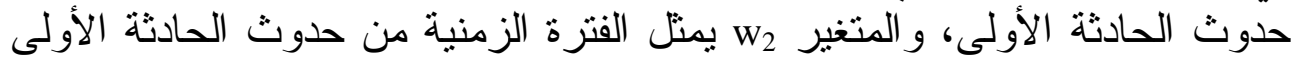

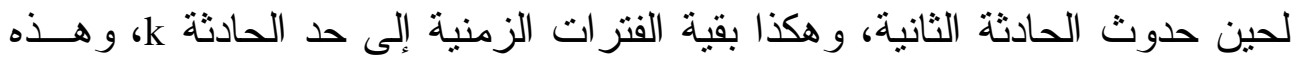

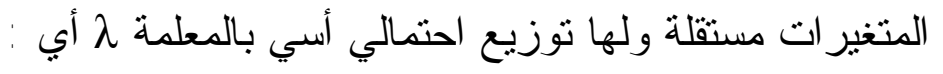

$$
p\left(W_{\mathrm{K}}\right)=\lambda \mathrm{e}^{-\lambda W_{\mathrm{k}}} \quad \mathrm{w}_{\mathrm{k}} \geq 1 \quad \cdots
$$

و إن معدل التوزيع الأسي (أوقات الوصول البينية)كما هو معلوم 1/2 في حين يكون تباينه

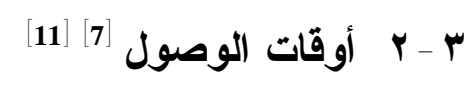

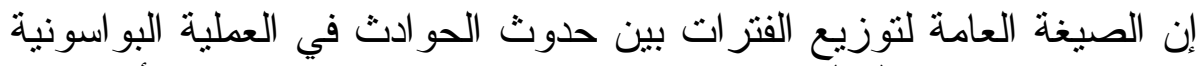

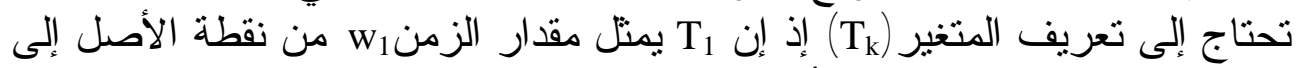

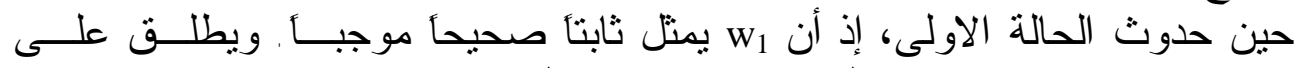

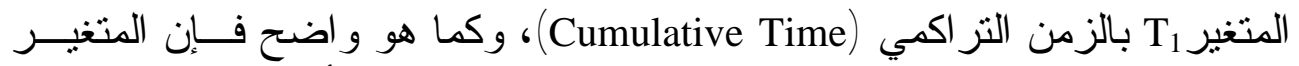

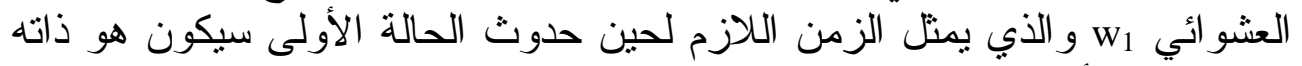

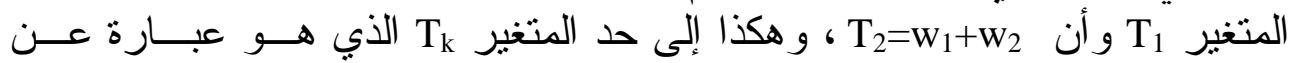

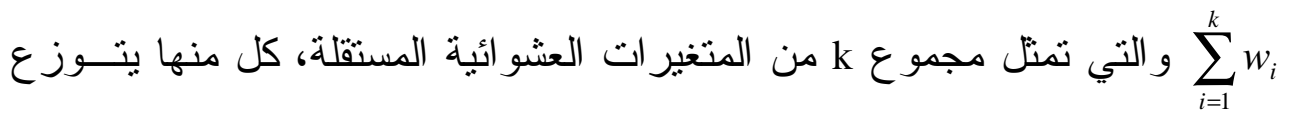




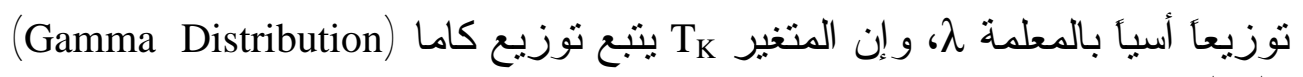

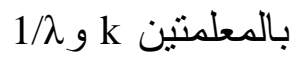
$p\left(T_{k}\right)=\frac{\lambda^{K}}{(K-1) !} T_{k}^{k-1} \mathrm{e}^{-\lambda T_{K}} \quad \mathrm{~T}_{\mathrm{k}} \geq 0 \quad \ldots \quad$ (2.3)

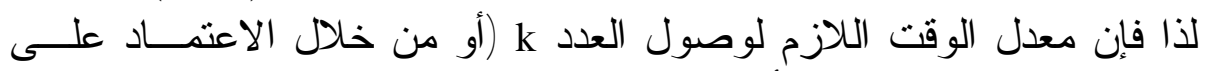

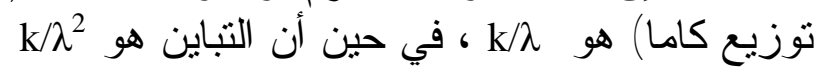
r. أ تحليل بيز لعمليات بواسون

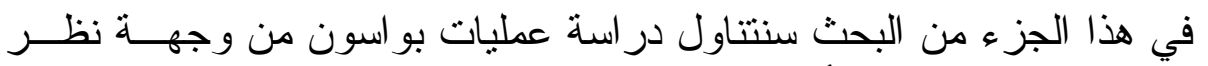

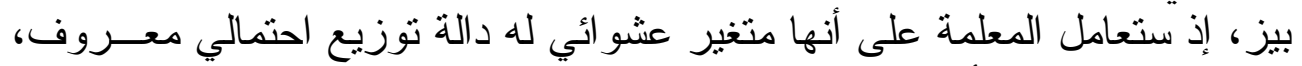

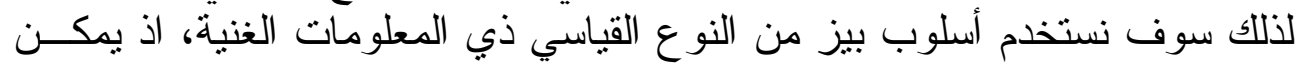

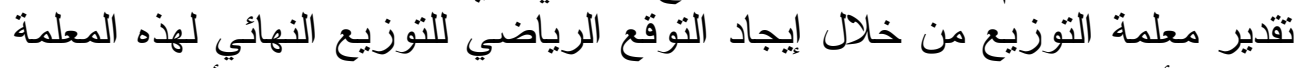

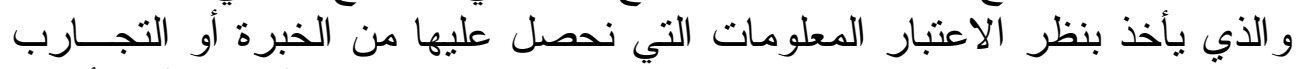

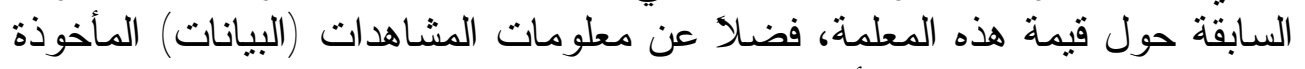

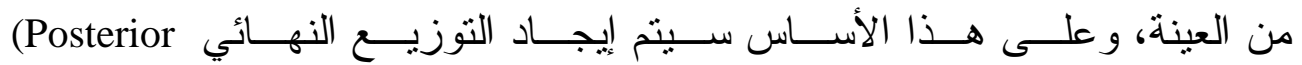

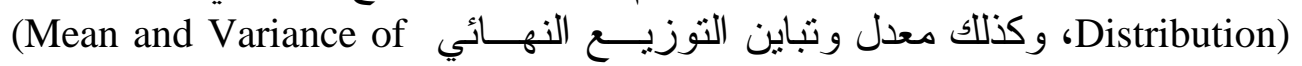

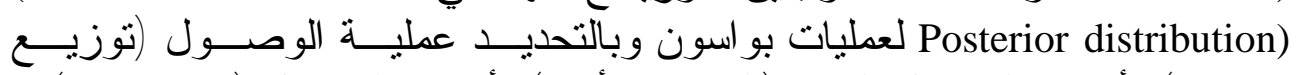
بو اسون)، أوقات الوصول البينية (التوزيع الأسي)، أوقات الوصول (توزيع كاما).

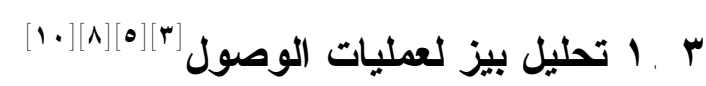

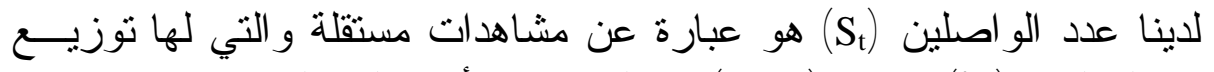

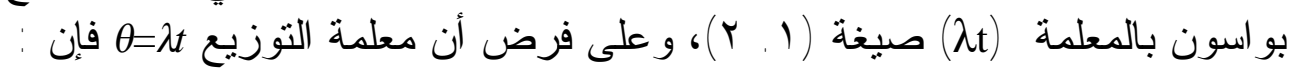
$P\left(S_{t} ; \theta\right)=\frac{(\theta)^{s_{t}} \mathrm{e}^{-\theta}}{S_{t} !} \quad ; \quad \mathrm{s}_{\mathrm{t}}=0,1,2, \ldots$ $\ln P\left(S_{t} ; \theta\right)=S_{t} \ln \theta-\theta-\ln S_{t}$

$$
\begin{aligned}
\frac{\partial \ln P\left(S_{t} ; \theta\right)}{\partial \theta}= & \frac{S_{t}}{\theta}-1 \\
\frac{\partial^{2} \ln P\left(S_{t} ; \theta\right)}{\partial \theta^{2}}= & -\frac{S_{t}}{\theta^{2}} \\
& , \theta \text { ومن خلال استخدام صيغة معلومات فيشر حول المعلمة }
\end{aligned}
$$




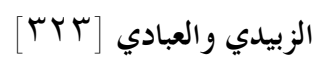

$$
\begin{aligned}
& f_{s_{t}}(\theta)=-E\left[\frac{\partial^{2} \ln p\left(s_{t} ; \theta\right)}{\partial \theta^{2}}\right] \\
& f_{s_{t}}(\theta)=-E\left[-\frac{S_{t}}{\theta^{2}}\right]=\frac{1}{\theta^{2}} E\left(s_{t}\right)=\frac{1}{\theta^{2}} \cdot \theta=\frac{1}{\theta}
\end{aligned}
$$

وباستخدام قانون جيفريز يمكن الحصول على التوزيع الأولي

$$
\text { : القياسي لتوزيع بو اسون وكما يأتيون Distribution) }
$$

$$
P(\theta)=\sqrt{f_{s_{t}}(\theta)}=\theta^{-\frac{1}{2}}
$$

ولدينا دالة التزجيح لتوزيع بو اسون كما يأتي :

$p\left(s_{t} / \theta\right) \propto \mathrm{e}^{-n \theta} \theta^{\sum_{i=1}^{n} s_{t_{i}}}$

$$
p\left(\theta / s_{t}\right) \propto p(\theta) \cdot p\left(s_{t} / \theta\right)
$$

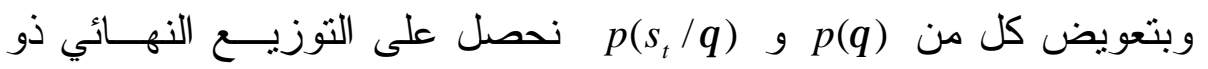

المعلومات القليلة وكما يأتي :

$$
\begin{gathered}
p\left(\theta / s_{t}\right) \propto \theta^{-\frac{1}{2}} \mathrm{e}^{-n \theta} \theta^{\sum_{i=1}^{n} s_{s_{i}}} \\
=\theta^{\sum_{i=1}^{n} s_{t_{i}}-\frac{1}{2}} \mathrm{e}^{-n \theta}
\end{gathered}
$$

$$
p\left(\theta / s_{t}\right) \propto \theta^{\mathrm{a}-1} \mathrm{e}^{-\mathrm{b} \theta}
$$

$a=\sum_{i=1}^{n} s_{t_{i}}+\frac{1}{2} \quad ; \quad \mathrm{b}=\mathrm{n}$

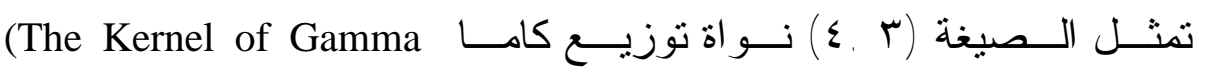
: Distribution) $\left(\theta / s_{t}\right) \sim \mathrm{G}(\mathrm{a}, \mathrm{b})$

ويمكن إيجاد النوزيع النهائي لعدد الواصلين باستخدام النوزيــع الأولـي ذي إني

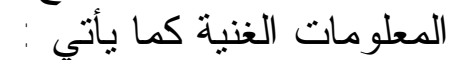

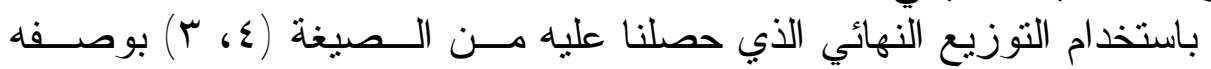
توزيعاً أولياً بالمعلمات $p(\theta) \infty \theta^{a_{0}-1} \mathrm{e}^{-b_{0} \theta}$ 
$\theta \sim \mathrm{G}\left(\mathrm{a}_{0}, \mathrm{~b}_{0}\right)$

ولدينا دالة التزجيح (s) نظرية بيز نحصل على التوزيع النهائي للمعلمة $\theta$ وكما يأتي : $p\left(\theta / s_{t}\right) \propto \theta^{\mathrm{a}_{0}-1} \mathrm{e}^{-\mathrm{b}_{0} \theta} \mathrm{e}^{-\mathrm{n} \theta} \theta^{\sum_{\mathrm{i}=1}^{\mathrm{n}} s_{t_{i}}}$

$$
=\theta{ }^{\left(a_{0}+\sum_{i=1}^{n} s_{s_{i}}\right)-1} e^{-\left(b_{0}+n\right) \theta}
$$

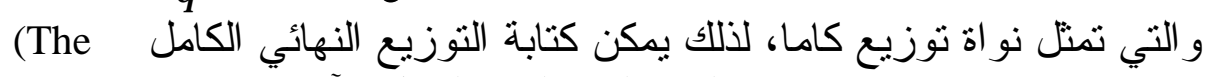

لعدد الو اصلين بالثكل الآتي : Complete Posterior Distribution )

$p\left(\theta / s_{t}\right)=\frac{\left(b_{0}+\mathrm{n}\right)^{\left(\mathrm{a}_{0}+\sum_{\mathrm{i}=1}^{\mathrm{n}} \mathrm{s}_{\mathrm{t}_{\mathrm{i}}}\right)}}{\Gamma\left(\mathrm{a}_{0}+\sum_{\mathrm{i}=1}^{\mathrm{n}} \mathrm{s}_{\mathrm{t}_{\mathrm{i}}}\right)} \theta^{\left(\mathrm{a}_{0}+\sum_{i=1}^{n} s_{i_{i}}\right)-1} \mathrm{e}^{-\left(\mathrm{a}_{0}+\mathrm{n}\right) \theta} \quad \ldots$

وبالتعويض عن قيمة المعلمة $\theta$ فإن :

$\left(\lambda t / s_{t}\right) \sim \mathrm{G}\left(\mathrm{a}_{0}+\sum_{i=1}^{n} s_{t_{i}}, b_{0}+n\right)$

معدل التوزيع النهائي لعدد الو اصلين بالاعتماد على معدل توزيع كاما يكون

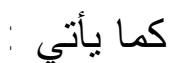

$\mu=\mathrm{E}\left(\lambda \mathrm{t} / s_{t}\right)=\frac{\mathrm{a}_{0}+\sum_{i=1}^{\mathrm{n}} s_{t_{i}}}{\mathrm{~b}_{0}+\mathrm{n}}$

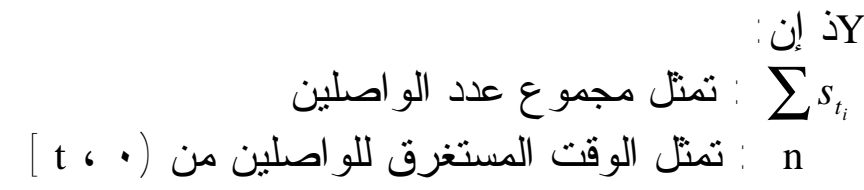

في حين يكون تباين التوزيع النهائي لعدد الو اصلين كما يأتي :

$\operatorname{var}\left(\lambda \mathrm{t} / s_{t}\right)=\frac{{ }_{0}+\sum_{i=1}^{\mathrm{n}} s_{t_{i}}}{\left(\mathrm{~b}_{0}+\mathrm{n}\right)^{2}} \quad \ldots$

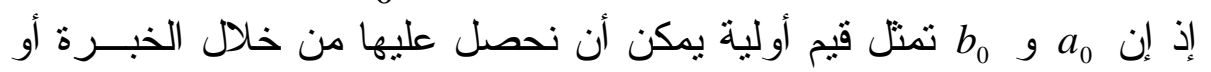

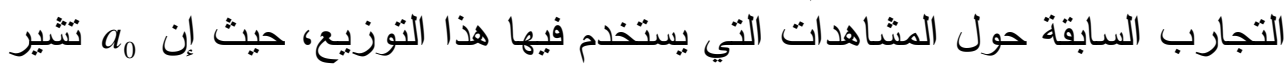

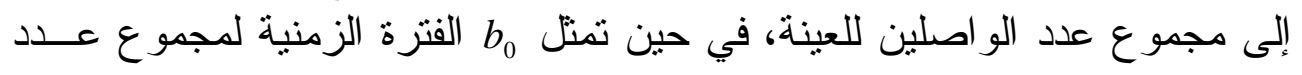

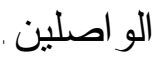


الزبيدي والعبادي [ro

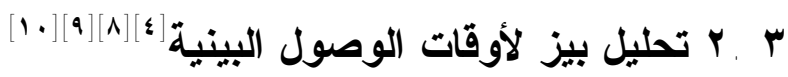

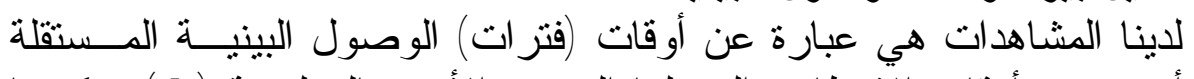

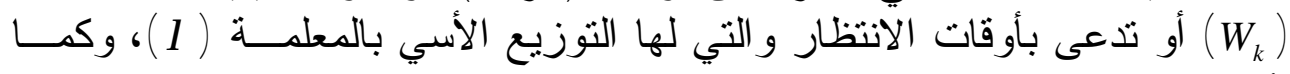
يأتي:

$p\left(W_{k} ; \lambda\right)=\lambda \mathrm{e}^{-\lambda w_{k}} \quad ; \quad W_{k} \geq 0 \quad \ldots$

$\ln p\left(W_{k} ; \lambda\right)=\ln \lambda-\lambda W_{k}$

$\frac{\partial \ln p\left(W_{k} ; \lambda\right)}{\partial \lambda}=\frac{1}{\lambda}-W_{k}$

$\frac{\partial^{2} \ln p\left(W_{k} ; \lambda\right)}{\partial \lambda^{2}}=\frac{-1}{\lambda^{2}}$

ومن خلال استخدام صيغة معلومات فيشر حول المعلمة $\lambda$ نحصل على :

$f_{w_{k}}(\lambda)=\frac{1}{\lambda^{2}}$

وباستخدام قانون جيفريز يمكن الحصول على التوزيع الأولي القياسي للتوزيع

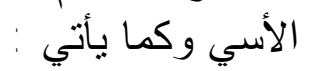

$p(\lambda)=\lambda^{-1}$

ولدنيا دالة الترجيح للتوزيع الأسي كما يأتي :

$p\left(W_{k} / \lambda\right)=\lambda^{n} \mathrm{e}^{-\lambda \sum_{i=1}^{n} w_{k_{i}}} \ldots$

و بالتعويض بنظرية بيز نحصل على النوزيع النهائي ذي المعلومات القليلة

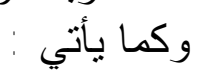
$p\left(\lambda / W_{k}\right) \propto \lambda^{-1} \lambda^{n} \mathrm{e}^{-\lambda \sum_{\mathrm{i}=1}^{\mathrm{n}} \mathrm{w}_{\mathrm{k}_{\mathrm{i}}}}$

$$
=\lambda^{n-1} \mathrm{e}^{-\lambda \sum_{i=1}^{n} w_{k_{i}}}
$$

$$
p\left(\lambda / W_{k}\right) \propto \lambda^{\mathrm{a}-1} \mathrm{e}^{-\mathrm{b} \lambda} \quad \ldots
$$




$$
a=n \quad ; \quad \mathrm{b}=\sum_{\mathrm{i}=1}^{\mathrm{n}} \mathrm{w}_{\mathrm{k}_{\mathrm{i}}}
$$

$\left(\lambda / W_{K}\right) \sim \mathrm{G}(\mathrm{a}, \mathrm{b})$

$$
\text { تمنل الصيغة (1) (1) نواة توزيع كاما، أي أن : }
$$

ويمكن إيجاد التوزيع النهائي لأوقات الوصول البينية باستخدام التوزيع الأولي

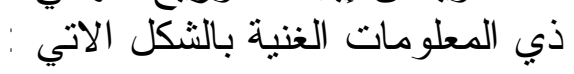

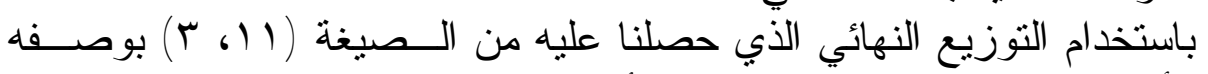
توزيعا أولياً بالمعلمات (a $p(\lambda) \propto \lambda^{\mathrm{a}_{0}-1} \mathrm{e}^{-\mathrm{b}_{0} \lambda}$

$\lambda \sim \mathrm{G}\left(\mathrm{a}_{0}, \mathrm{~b}_{0}\right)$

ولاينا دالة الترجيح للتوزيع الأسي من الصيغة ( • (r, و) وبتطبيق نظرية بيز

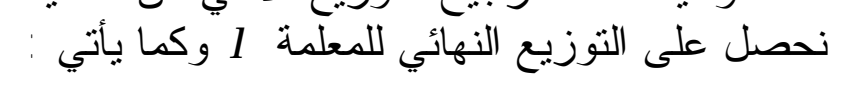
$p\left(\lambda / W_{k}\right) \propto \lambda^{a_{0}-1} \mathrm{e}^{-b_{0} \lambda} \lambda^{n} \mathrm{e}^{-\lambda \sum_{i=1}^{n} w_{k_{i}}}$

$$
=\lambda^{\left(a_{0}+n\right)-1} \mathrm{e}^{-\left(b_{0}+\sum_{\mathrm{i}=1}^{\mathrm{n}} \mathrm{w}_{\mathrm{k}_{\mathrm{i}}}\right)}
$$

و التي تمتل نواة نوزيع كاما، لذلك يمكن كتابة التوزيع النهائي الكامل بالثنكل

$p\left(\lambda / W_{k}\right)=\frac{\left(b_{0}+\sum_{i=1}^{n} w_{k}\right)^{\left(a_{0}+n\right)}}{\Gamma\left(a_{0}+n\right)} \lambda^{\left(a_{0}+n\right)-1} \mathrm{e}^{-\left(b_{0}+\sum_{i=1}^{n} w_{k}\right) \lambda} \ldots$

$\left(\lambda / W_{k}\right) \sim \mathrm{G}\left(\mathrm{a}_{0}+n, \mathrm{~b}_{0}+\sum_{i=1}^{n} w_{k_{i}}\right)$

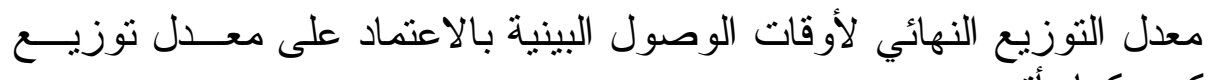

كاما يكون كما يأتي :

$$
\mu=E\left(\lambda / W_{k}\right)=\frac{a_{0}+n}{b_{0}+\sum_{i=1}^{n} w_{k_{i}}}
$$




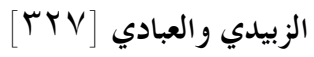

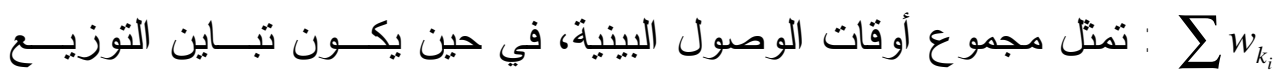
النهائي لأوقات الوصول البينية كما يأني:

$$
\operatorname{Var}=E\left(\lambda / W_{k}\right)=\frac{a_{0}+n}{\left(b_{0}+\sum_{i=1}^{n} w_{k_{i}}\right)^{2}} \quad \cdots
$$

إذ إن

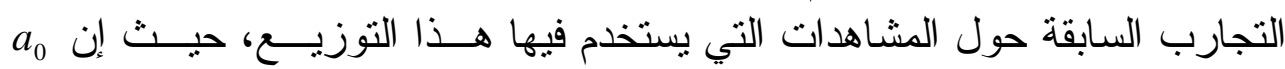

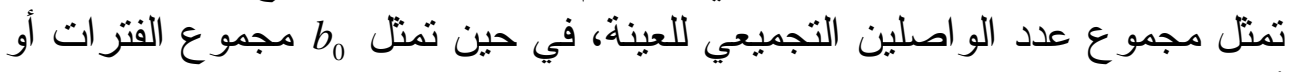
أوقات الوصول البينية للعينة.

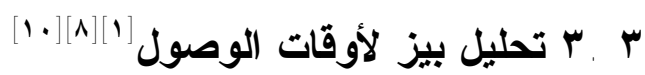

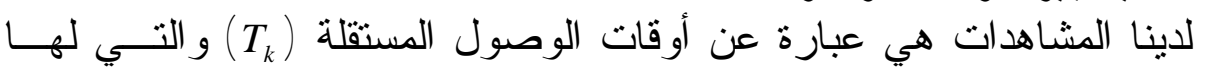

$$
\text { توزيع كاما بالمعالم ( , k }
$$

$p\left(T_{k}\right)=\frac{\lambda^{k}}{(k-1)} T_{k}^{k-1} \mathrm{e}^{-\lambda T_{\mathrm{K}}} \quad ; \quad \mathrm{T}_{\mathrm{k}} \geq 0 \quad \ldots$

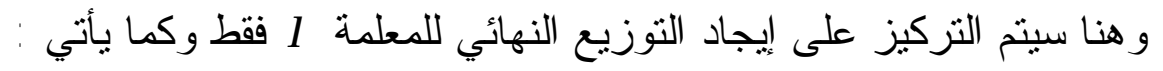

$\ln p\left(T_{k} ; \lambda\right)=k \ln \lambda+\ln \left[\frac{T_{k}^{k-1}}{(k-1)}\right]-\lambda T_{k}$

$\frac{\partial \ln p\left(T_{k} ; \lambda\right)}{\partial \lambda}=\frac{k}{\lambda}-T_{k}$

$\frac{\partial^{2} \ln p\left(T_{k} ; \lambda\right)}{\partial \lambda^{2}}=\frac{-k}{\lambda}$

ومن خلال استخدام صيغة معلومات فيشر حول المعلمة ح، لدينا : $f_{T_{k}}(\lambda)=\frac{k}{\lambda^{2}}$

وباستخدام قانون جيفريز يمكن الحصول على التوزيع الأولي القياسي لنوزيع

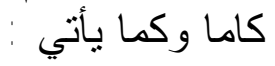

$$
p(\lambda) \propto \lambda^{-1}
$$

ولدينا دالة التزجيح لتوزيع كاما كما يأني : 


$$
p\left(T_{k} / \lambda\right) \propto \lambda^{\mathrm{nk}} \mathrm{e}^{-\lambda \sum_{\mathrm{i}=1}^{\mathrm{n}} \mathrm{T}_{\mathrm{k}_{\mathrm{i}}}}
$$

وبالتعويض بنظرية بيز نحصل على التوزيع النهائي ذي المعلومات القليلـــة

$$
\begin{gathered}
p\left(\lambda / T_{k}\right) \propto \lambda^{-1} \lambda^{\mathrm{nk}} \mathrm{e}^{-\lambda \sum_{\mathrm{i}=1}^{\mathrm{n}} \mathrm{T}_{\mathrm{k}_{\mathrm{i}}}} \\
=\lambda^{\mathrm{nk}-1} \mathrm{e}^{-\lambda \sum_{\mathrm{i}=1}^{\mathrm{n}} \mathrm{T}_{\mathrm{k}_{\mathrm{i}}}} \\
p\left(\lambda / T_{k}\right) \propto \lambda^{\mathrm{a}-1} \mathrm{e}^{-\mathrm{b} \lambda}
\end{gathered}
$$

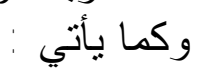

$$
\begin{aligned}
& a=n k \quad ; \quad \mathrm{b}=\sum_{\mathrm{i}=1}^{\mathrm{n}} \mathrm{T}_{\mathrm{k}_{\mathrm{i}}} \\
& \left(\lambda / T_{k}\right) \sim \mathrm{G}(\mathrm{a}, \mathrm{b}) \\
& \text { تمنل الصيغة (r, 1 ) نواة توزيع كاما، أي أن : }
\end{aligned}
$$$$
\text { ويمكن إيجاد التوزيع النهائي لأوقات الوصول باستخدام التوزيع الأولــي ذي الـي }
$$

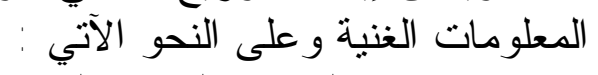

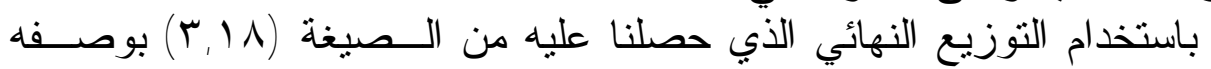

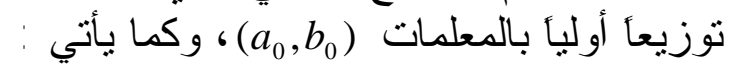$$
p(\lambda) \propto \lambda^{\mathrm{a}_{0}-1} \mathrm{e}^{-\mathrm{b}_{0} \lambda}
$$

$$
\lambda \sim G\left(a_{0}, b_{0}\right)
$$

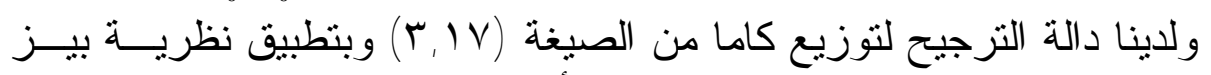
نحصل على التوزيع النهائي للمعلمة $\lambda$ وكما يأتي : $p\left(\lambda / T_{k}\right) \propto \lambda^{\mathrm{a}_{0}-1} \mathrm{e}^{-\mathrm{b}_{0} \lambda} \lambda^{n k} \mathrm{e}^{-\lambda \sum_{i=1}^{n} T_{k_{i}}}$

$$
=\lambda^{\left(a_{0}+n k\right)-1} \mathrm{e}^{-\left(b_{0}+\sum_{i=1}^{n} T_{k_{i}}\right) \lambda}
$$

و التي تمتل نو اة توزيع كاما لذللك يمكن كتابة التوزيع النهائي الكامل بالصيغة 
$\left(\lambda / T_{k}\right)=\frac{\left(b_{0}+\sum_{i=1}^{n} T_{k_{i}}\right)^{\left(a_{0}+n k\right)}}{\Gamma\left(a_{0}+n k\right)} \lambda^{\left(a_{0}+n k\right)-1} \mathrm{e}^{-\left(b_{0}+\sum_{i=1}^{n} T_{k_{i}}\right) \lambda} \quad \ldots$

$\left(\lambda / T_{k}\right) \sim G\left(a_{0}+n k, \mathrm{~b}_{0}+\sum_{i=1}^{n} T_{k_{i}}\right)$

معدل النوزيع النهائي لأوقات الوصول بالاعتماد على معدل توزيع كاما يكون

$$
\mu=E\left(\lambda / T_{k}\right)=\frac{a_{0}+n k}{b_{0}+\sum_{i=1}^{n} T_{k_{i}}}
$$

ST الوصول كما يأتي :

$$
\operatorname{Var}\left(\lambda / T_{k}\right)=\frac{a_{0}+n k}{\left(b_{0}+\sum_{i=1}^{n} T_{k_{i}}\right)^{2}} \quad \ldots
$$

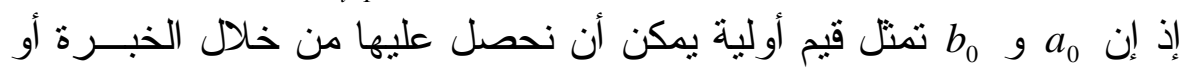

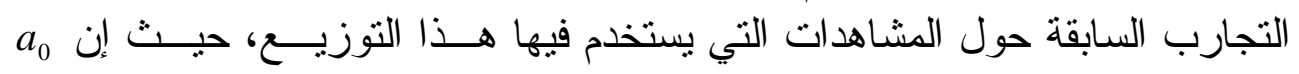

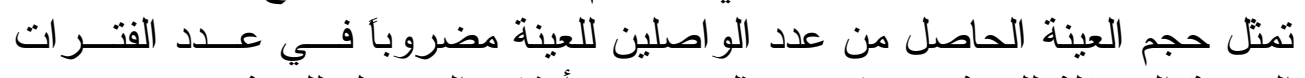

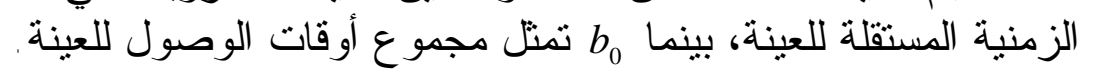

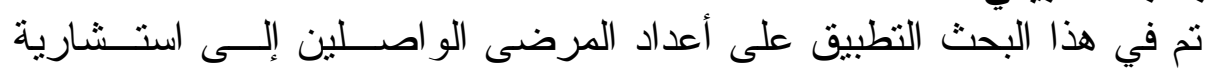

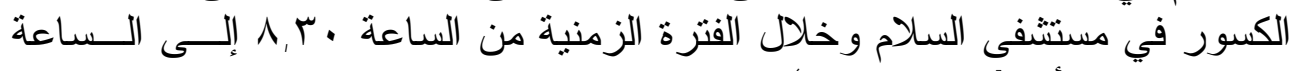

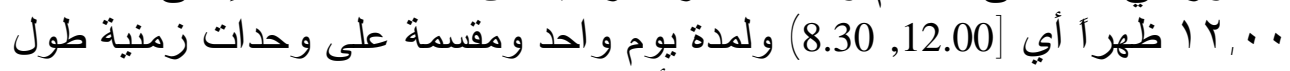

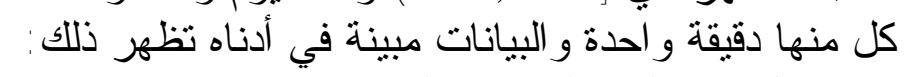
حيث تتبع توزيع بو اسون.

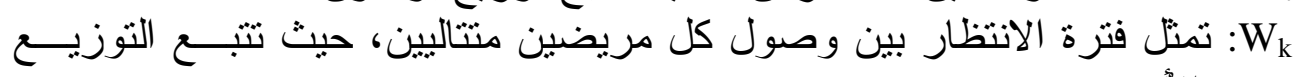

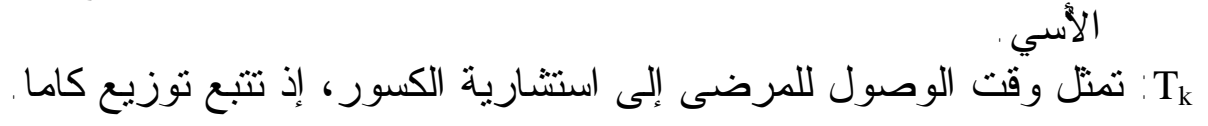




\begin{tabular}{|c|c|c|c|}
\hline \multicolumn{4}{|c|}{ في استشار وأوقات الوصول ألوصول للمرضى على التوالي } \\
\hline النسلسل & $\begin{array}{l}\text { Number } \\
\text { Arrived }\end{array}$ & Waiting time & Time arrived \\
\hline 1 & 1 & 10 & 10 \\
\hline 2 & 5 & 19 & 29 \\
\hline 3 & 11 & 15 & 44 \\
\hline 4 & 16 & 11 & 55 \\
\hline 5 & 20 & 8 & 63 \\
\hline 6 & 24 & 8 & 71 \\
\hline 7 & 28 & 8 & 79 \\
\hline 8 & 30 & 6 & 85 \\
\hline 9 & 32 & 13 & 98 \\
\hline 10 & 39 & 16 & 114 \\
\hline 11 & 43 & 19 & 133 \\
\hline 12 & 50 & 40 & 173 \\
\hline 13 & 51 & 10 & 183 \\
\hline 14 & 53 & 5 & 188 \\
\hline$\overline{15}$ & 55 & 5 & 193 \\
\hline 16 & 56 & 10 & 203 \\
\hline 17 & 59 & 7 & 210 \\
\hline $\bar{\sum}$ & 573 & 210 & 1931 \\
\hline
\end{tabular}

(*) - (*) الجدول من أعداد الباحثين 1 - التطبيق العملي لعمليات بواسون باستخدام الإحصاء الكلاســيكي -عمليــات

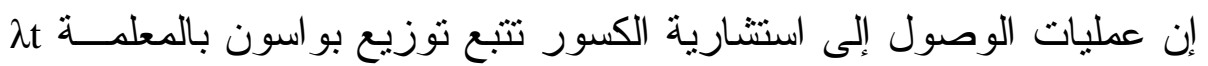

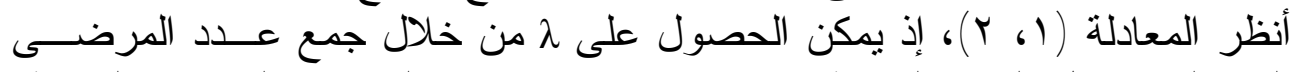
الو اصلين خلال الفترة الزمنية (8.30,12.00] وتقسيمها على عدى الفي عد الوحدات الزمنية، $\lambda=\frac{S_{t}}{t}=\frac{59}{210}=0.2809$

$\mu=\lambda \mathrm{t}=(0.2809)(210)=59$

فإن معدل الوصول هو :

Variance $=\mu=59$

وبما أن تباين نوزيع بو اسون يساوي معدله، أب أن : 


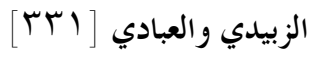

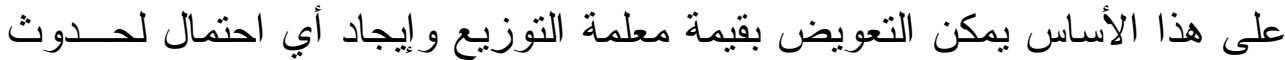

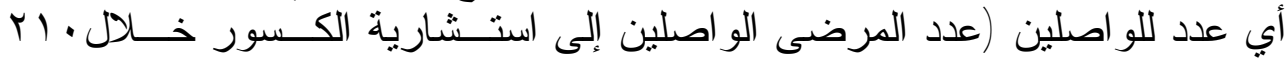

\section{- أوقات الوصول البينية}

نحصل على أوقات الوصول البينية (فترات الوصول) الوصلية من خلال ما يأتي:

$$
\text { لدينا أوقات الوصول التي نحصل عليها من عدد الو الصلين وهي الوني }
$$

$\mathrm{T}_{0}=0, \mathrm{~T}_{1}=10, \mathrm{~T}_{2}=29$ $T_{17}=210$

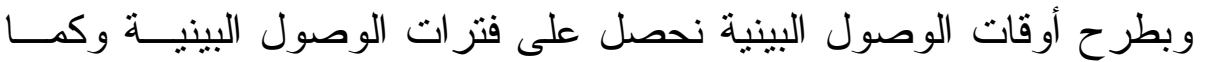

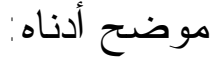

$\mathrm{W}_{1}=\mathrm{T}_{1}-\mathrm{T}_{0}=10-0=10$
$\mathrm{~W}_{2}=\mathrm{T}_{2}-\mathrm{T}_{1}=29-10=19$
$\mathrm{~W}_{3}=\mathrm{T}_{3}-\mathrm{T}_{2}=44-29=15$

$\mathrm{W}_{17}=\mathrm{T}_{17-} \mathrm{T}_{16}=7$

$\sum_{i=1}^{17} W_{i}=t=210$

إذ إن إن $\sum_{i=1}^{17} W_{i}$ الأسي، ودالة الكثافة الاحتمالية لأوقات الوصول البينية (التوزيع الأسي) هي :

$$
p\left(w_{k}\right)=\lambda \mathrm{e}^{-w_{k}}=(0.2809) \mathrm{e}^{-0.2809 w_{k}} \quad W_{k} \geq 1
$$

ومعدل هذا التوزيع (معدل الفترات الزمنية لوصول مريض و احد) هو : $\mu=\frac{1}{\lambda}=\frac{1}{0.2809}=3.5599$ وتباين هذا التوزيع (أوقات الوصول البينية) هو : $\operatorname{var}\left(W_{K}\right)=\frac{1}{\lambda^{2}}=\frac{1}{(0.2809)^{2}}=12.6735$

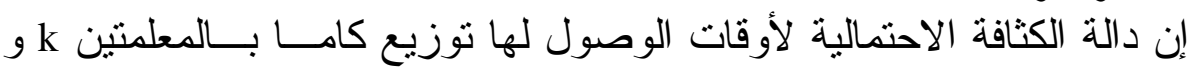

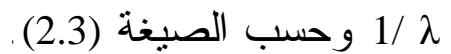
لذا فإن معدل هذا التوزيع (أوقات الوصول) هو : 


$$
\mu=\frac{k}{\lambda}=\frac{59}{0.2809}=210.039
$$

$$
\text { وتباين وقت الوصول }
$$

$$
\operatorname{var}\left(T_{k}\right)=\frac{k}{\lambda^{2}}=\frac{59}{(0.2809)^{2}}=747.7364
$$

r - ـ ـالتطبيق العملي لعمليات بواسون باستخدام أسلوب بيز

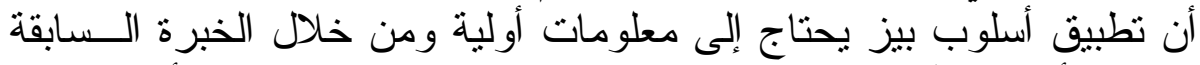

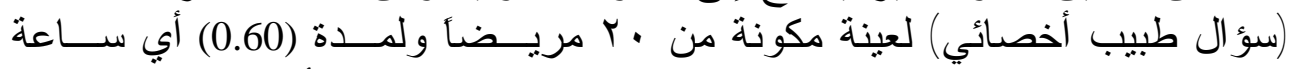

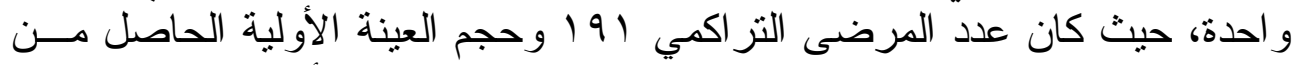

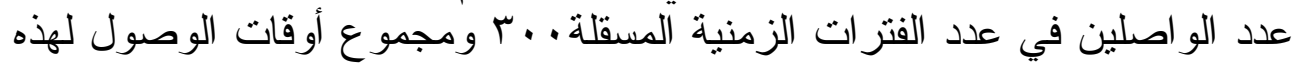

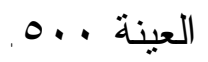

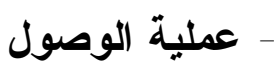

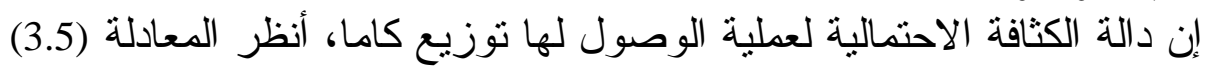
ولتقدير متوسط وتباين هذا التوزيع بالاعتماد على المعادلتين (3.6) و (3.7): $a_{0}=20$

$b_{0}=60$

$$
\lambda=\frac{a_{0}+\sum_{i=0}^{n} s_{t_{i}}}{\left(b_{0}+n\right)}=\frac{20+59}{(60+210)}=0.29259
$$

$$
\mu=\lambda t=(0.29259)(210)=61.44444 \approx 61
$$

$\operatorname{var}\left(\lambda t / s_{t}\right)=\frac{a_{0}+\sum_{i=1}^{n} s_{t_{i}}}{\left(b_{0}+n\right)^{2}}=\frac{20+59}{(60+210)^{2}}=0.00133$

$\operatorname{Var}(\lambda t)=t^{2} \operatorname{var}(\lambda)=58.67901$

$$
\text { - أوقات الوصول البينية }
$$

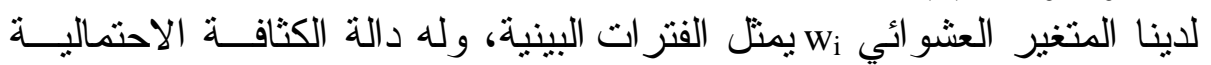

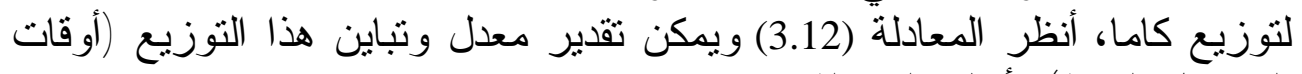
الوصول البينية)، أنظر المعادلة (3.13). 


$$
\begin{aligned}
& a_{0}=191 \\
& b_{0}=60 \\
& n=573 \\
& \sum w_{k}=210 \\
& \mu=E\left(\lambda / w_{k}\right)=\frac{191+573}{60+210}=2.82962
\end{aligned}
$$$$
\text { ونحصل على التباين بالاعتماد على المعادلة (3.14) إذ إن : }
$$

$\operatorname{Var}\left(\lambda / w_{k}\right)=\frac{191+573}{(60+210)^{2}}=0.01048$

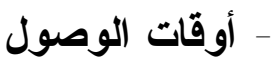

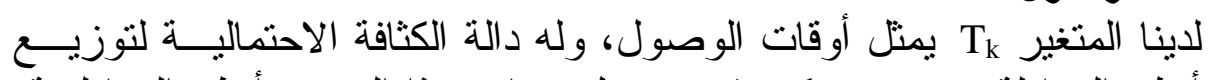

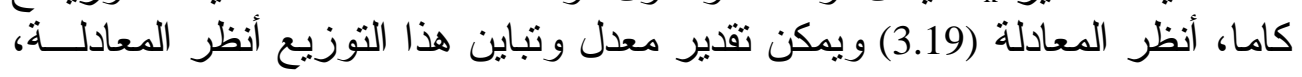

$$
\begin{aligned}
& a_{0}=300 \\
& b_{0}=500
\end{aligned}
$$

$$
\mu=E\left(\lambda / T_{k}\right)=\frac{300+59 * 17}{500+1931}=0.53599
$$

ونحصل على التباين بالاعتماد على المعادلة (3.21)

$\operatorname{Var}\left(\lambda / T_{k}\right)=\frac{300+59 * 17}{(500+1931)^{2}}=0.00022$

من خلال النتائج المذكورة آنفأ نلاحظ أن هناللك اختلافأ كبير آ بين النتائج التي التي

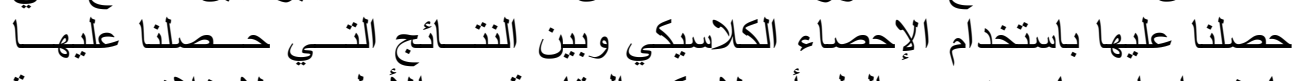

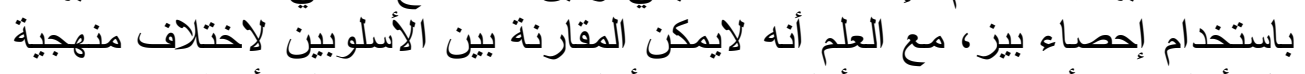

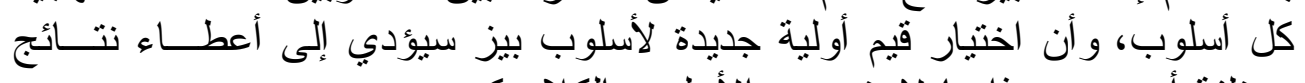
مختلفة أخرى، و هذا ما لا يتضمنه الأسلوب الكلاسيكي.

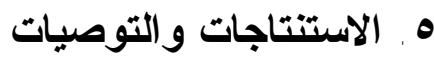

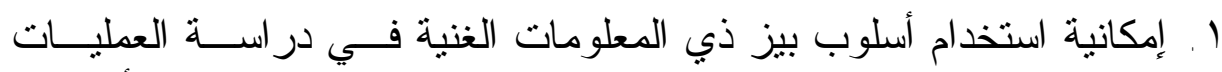

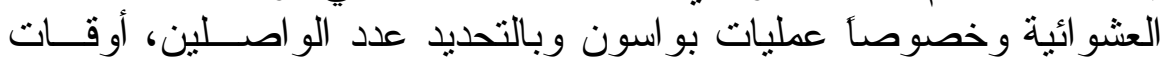
الوصول البينية، وأوقات الوصول ولئول 


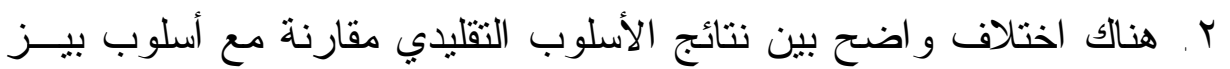

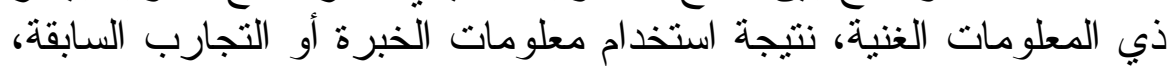

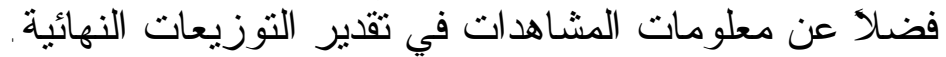

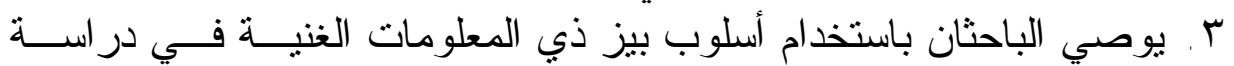

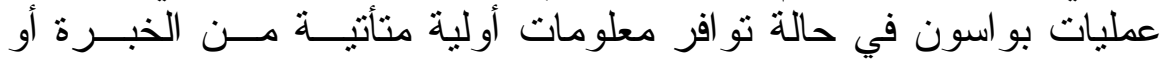
التجارب السابقة حول هذه العمليات.

\section{ثانياً - المراجع باللغة العربية}

1. Basawa,I.V,and Prakasa Rao ;B.L, 1980, Statistical Inference for Stochastic Processes, Academic Process,London.

2. Box and Tiao, 1973, Bayesian Inference in statistical Analysis, Addison-wesley publishing company California;London.

3. Jayanta, K; Mohan, D; Tapas, S, 2006, An Introduction to Bayesian Analysis; springer;USA

4. Jefferys,S.H, 1961, Theory of Probability ;clarenden press,oxford,London.

5. Mooney,D.D, and Swift ,R.J, 1999, Acourse in Mathematical Modeling "the Mathematical Association of American ;USA. 